\title{
Spectrum, distance spectrum, and Wiener index of wreath products of complete graphs
}

\author{
Alfredo Donno * \\ Università degli Studi Niccolò Cusano \\ via Don Carlo Gnocchi 3, 00166 Roma, Italia
}

Received 31 May 2016, accepted 16 December 2016, published online 3 March 2017

\begin{abstract}
We describe the adjacency matrix and the distance matrix of the wreath product of two complete graphs, and we give an explicit computation of their spectra. As an application, we deduce the spectrum of the transition matrix of the Lamplighter random walk over a complete base graph, with a complete color graph. Finally, an explicit computation of the Wiener index is given.
\end{abstract}

Keywords: Wreath product of complete graphs, adjacency matrix, distance matrix, spectrum, distance spectrum, Wiener index.

Math. Subj. Class.: 05C12, 05C50, 05C76, 05C81, $15 A 69$

\section{Introduction}

The construction of new graphs starting from smaller factor graphs is a very natural and fruitful technique, largely developed in literature for its theoretical interest in several branches of Mathematics - Algebra, Combinatorics, Probability, Harmonic Analysis - but also for its practical applications. Among the standard products we find, for instance, the Cartesian product, the direct product, the strong product, the lexicographic product [22, 23, 30, 31]. More recently, the zig-zag product was introduced [29], in order to produce expanders of constant degree and arbitrary size; in [10,14], some combinatorial and topological properties of such products, as well as connections with random walks, have been investigated.

It is worth mentioning that many of these constructions play an important role in Geometric Group Theory, since it turns out that, when applied to Cayley graphs of two finite groups, they provide the Cayley graph of an appropriate product of these groups (see [1],

* Telephone: +3906 45678356, Fax: +3906 45678379

E-mail address: alfredo.donno@unicusano.it (Alfredo Donno) 
where this correspondence is shown for zig-zag products, or [15], for the case of wreath and generalized wreath products).

Spectral properties of graph products have been object of an intensive study in the last decades, both for their algebraic and combinatorial interest, and for applications to Probability, Computer Science, and Mathematical Chemistry. The spectrum of a graph is defined as the spectrum of its adjacency matrix; similarly, the distance spectrum of a graph is defined to be the spectrum of its distance matrix (see Section 2). The reader can refer, for instance, to the monograph [5] for an exhaustive treatment of spectra of graphs. We also want to mention the papers [24, 25, 34], where the distance spectrum of some graph compositions has been studied.

A related topic of research is the Wiener index, which is defined as the sum of the distances between all the unordered pairs of vertices of the graph. This index was introduced by Wiener [36] and, due to the wide range of applications, it is nowadays largely studied. In particular, it is one of the most frequently used topological indices in mathematical chemistry, as molecules can be represented by means of undirected graphs. For this reason, it has a strong correlation with many physical and chemical properties of molecular compounds, whose properties do not only depend on their chemical formula, but also on their molecular structure [13]. There exists a wide range of fields such as communication, facility location, cryptology, architecture where the Wiener index of a graph is of great interest. A large number of papers is devoted to the study of the Wiener index of graphs, sequences of graphs, products of graphs. In [12] the Wiener index of trees is investigated. In [16] the Wiener index and the related Hosoya polynomial are studied for a family of circulant graphs. See also the paper [9], where the Wiener index is studied on an increasing sequence of finite graphs, introduced in [6], and whose limit graphs have been studied in [7], which approximates the Sierpiński carpet fractal. In [17, 18] the study of Wiener index is developed for some graph compositions.

In the present paper, we focus our attention on a different kind of graph product known in literature, namely the wreath product of two graphs (see Definition 2.1). This construction is nowadays largely studied, and different generalizations have been introduced $[15,19]$. Notice that this construction is interesting not only from an algebraic and combinatorial point of view, but also for its connection with Geometric group theory and Probability, via the notions of Lamplighter group and Lamplighter random walk (see, for instance, [3, 21, 32, 33, 37]). Notice that in a previous paper joint with D. D'Angeli [8], we introduced a matrix operation, called wreath product of matrices (recalled in Definition 2.2), which is a matrix analogue of the wreath product of graphs, since it provides the adjacency matrix of the wreath product of two graphs, when applied to the adjacency matrices of the factors (Theorem 2.3 below).

Let us denote by $K_{n}$ the complete graph on $n$ vertices. In this paper, the wreath product $K_{n} \prec K_{m}$ is studied. In Proposition 3.1, we describe in detail distances in $K_{n} \prec K_{m}$, and we deduce its diameter in Corollary 3.2. Moreover, in Proposition 3.4 we show that the graph $K_{n} \prec K_{m}$ is not distance-regular. After describing in detail the adjacency matrix of the wreath product $K_{n}$ 々 $K_{m}$ of two complete graphs, we are able to explicitly compute its spectrum by using a reduction argument, allowing to reduce our computations to the study of the spectrum of smaller matrices, whose size is the cardinality of the vertex set of the first graph (Theorem 3.7); we then deduce the spectrum of the transition matrix of the Lamplighter random walk with base graph $K_{n}$ and color graph $K_{m}$ (Corollary 3.9). In Proposition 3.10, we provide the distance matrix of $K_{n} \imath K_{m}$, and its spectrum is determined 
in Theorem 3.11 by means of a second reduction argument. Finally, in Theorem 3.13, the Wiener index of the graph $K_{n} \prec K_{m}$ is computed.

Notice that the spectrum considered in the present paper concerns the "walk or switch" Lamplighter random walk. The analogous question for the so called "switch-walk-switch" Lamplighter random walk has been solved in [26, 27]. A common framework for such computations has been established in [20].

\section{Preliminaries}

Let $\mathcal{G}=(V, E)$ be a finite undirected graph, where $V$ denotes the vertex set, and $E$ is the edge set consisting of unordered pairs of type $\{u, v\}$, with $u, v \in V$. If $\{u, v\} \in E$, we say that the vertices $u$ and $v$ are adjacent in $\mathcal{G}$, and we use the notation $u \sim v$. A path in $\mathcal{G}$ is a sequence $u_{0}, u_{1}, \ldots, u_{\ell}$ of vertices such that $u_{i} \sim u_{i+1}$, for each $i=0, \ldots, \ell-1$. We say that such a path has length $\ell$. The graph is connected if, for every $u, v \in V$, there exists a path $u_{0}, u_{1}, \ldots, u_{\ell}$ in $\mathcal{G}$ such that $u_{0}=u$ and $u_{\ell}=v$. For a connected graph $\mathcal{G}$, we will denote by $d(u, v)$ the geodesic distance between the vertices $u$ and $v$, that is, the length of a minimal path in $\mathcal{G}$ joining $u$ and $v$. The diameter of $\mathcal{G}$ is then defined as $\operatorname{diam}(\mathcal{G})=\max _{u, v \in V}\{d(u, v)\}$.

Recall now that the adjacency matrix of an undirected graph $\mathcal{G}=(V, E)$ is the square matrix $A=\left(a_{u, v}\right)_{u, v \in V}$, indexed by the vertices of $\mathcal{G}$, whose entry $a_{u, v}$ equals the number of edges connecting $u$ and $v$. As the graph $\mathcal{G}$ is undirected, $A$ is a symmetric matrix and so all its eigenvalues are real. The spectrum of $\mathcal{G}$ is then defined as the spectrum of its adjacency matrix. The degree of a vertex $u \in V$ is defined as $\operatorname{deg}(u)=\sum_{v \in V} a_{u, v}$. In particular, we say that $G$ is regular of degree $d$, or $d$-regular, if $\operatorname{deg}(u)=d$, for each $u \in V$. In this case, the normalized adjacency matrix $A^{\prime}$ of $\mathcal{G}$ is obtained as $A^{\prime}=\frac{1}{d} A$.

We recall now the definition of wreath product of graphs.

Definition 2.1. Let $\mathcal{G}_{1}=\left(V_{1}, E_{1}\right)$ and $\mathcal{G}_{2}=\left(V_{2}, E_{2}\right)$ be two finite graphs. The wreath product $\mathcal{G}_{1} \prec \mathcal{G}_{2}$ is the graph with vertex set $V_{2}^{V_{1}} \times V_{1}=\left\{(f, v) \mid f: V_{1} \rightarrow V_{2}, v \in V_{1}\right\}$, where two vertices $(f, v)$ and $\left(f^{\prime}, v^{\prime}\right)$ are connected by an edge if:

(1) (edges of type I) either $v=v^{\prime}=: \bar{v}$ and $f(w)=f^{\prime}(w)$ for every $w \neq \bar{v}$, and $f(\bar{v}) \sim f^{\prime}(\bar{v})$ in $\mathcal{G}_{2} ;$

(2) (edges of type II) or $f(w)=f^{\prime}(w)$, for every $w \in V_{1}$, and $v \sim v^{\prime}$ in $\mathcal{G}_{1}$.

It follows from the definition that, if $\mathcal{G}_{1}$ is a regular graph on $n_{1}$ vertices with degree $d_{1}$ and $\mathcal{G}_{2}$ is regular graph on $n_{2}$ vertices with degree $d_{2}$, then the graph $\mathcal{G}_{1} \prec \mathcal{G}_{2}$ is a $\left(d_{1}+d_{2}\right)$ regular graph on $n_{1} n_{2}^{n_{1}}$ vertices.

It is a classical fact (see, for instance, [37]) that the simple random walk on the graph $\mathcal{G}_{1} \prec \mathcal{G}_{2}$ is the so called Lamplighter random walk, according to the following interpretation: suppose that at each vertex of $\mathcal{G}_{1}$ (the base graph) there is a lamp, whose possible states (or colors) are represented by the vertices of $\mathcal{G}_{2}$ (the color graph), so that the vertex $(f, v)$ of $\mathcal{G}_{1} \prec \mathcal{G}_{2}$ represents the configuration of the $\left|V_{1}\right|$ lamps at each vertex of $\mathcal{G}_{1}$ (for each vertex $u \in V_{1}$, the lamp at $u$ is in the state $f(u) \in V_{2}$ ), together with the position $v$ of a lamplighter walking on the graph $\mathcal{G}_{1}$. At each step, the lamplighter may either go to a neighbor of the current vertex $v$ and leave all lamps unchanged (this situation corresponds to edges of type II in $\mathcal{G}_{1} \prec \mathcal{G}_{2}$ ), or he may stay at the vertex $v \in \mathcal{G}_{1}$, but he changes the state of the lamp which is in $v$ to a neighbor state in $\mathcal{G}_{2}$ (this situation corresponds to edges of type I in $\mathcal{G}_{1} \prec \mathcal{G}_{2}$ ). For this reason, the wreath product $\mathcal{G}_{1} \prec \mathcal{G}_{2}$ is also called the Lamplighter 
graph, or Lamplighter product, with base graph $\mathcal{G}_{1}$ and color graph $\mathcal{G}_{2}$. Also notice that the model described above is often called "walk or switch" Lamplighter random walk.

It is worth mentioning that the wreath product of graphs represents a graph analogue of the classical wreath product of groups [28], as it turns out that the wreath product of the Cayley graphs of two finite groups is the Cayley graph of the wreath product of the groups, with a suitable choice of the generating sets. In the paper [15], this correspondence is proven in the more general context of generalized wreath products of graphs, inspired by the construction introduced in [2] for permutation groups. Also notice that in [19] a different notion of generalized wreath product of graphs is presented.

In the paper [8], the following matrix construction involving wreath products is introduced. Let $\mathcal{M}_{m \times n}(\mathbb{C})$ denote the set of matrices with $m$ rows and $n$ columns over the complex field, and let $I_{n}$ be the identity matrix of size $n$. We recall that the Kronecker product of two matrices $A=\left(a_{i j}\right)_{i=1, \ldots, m ; j=1, \ldots, n} \in \mathcal{M}_{m \times n}(\mathbb{C})$ and $B=\left(b_{h k}\right)_{h=1, \ldots, p ; k=1, \ldots, q} \in$ $\mathcal{M}_{p \times q}(\mathbb{C})$ is defined to be the $m p \times n q$ matrix

$$
A \otimes B=\left(\begin{array}{ccc}
a_{11} B & \cdots & a_{1 n} B \\
\vdots & \ddots & \vdots \\
a_{m 1} B & \cdots & a_{m n} B
\end{array}\right) .
$$

We denote by $A^{\otimes^{n}}$ the iterated Kronecker product $\underbrace{A \otimes \cdots \otimes A}_{n \text { times }}$, and we put $A^{\otimes^{0}}=1$.

Definition 2.2 ([8]). Let $A \in \mathcal{M}_{n \times n}(\mathbb{C})$ and $B \in \mathcal{M}_{m \times m}(\mathbb{C})$. For each $i=1, \ldots, n$, let $C_{i}=\left(c_{h k}\right)_{h, k=1, \ldots, n} \in \mathcal{M}_{n \times n}(\mathbb{C})$ be the matrix defined by

$$
c_{h k}= \begin{cases}1 & \text { if } h=k=i \\ 0 & \text { otherwise. }\end{cases}
$$

The wreath product of $A$ and $B$ is the square matrix of size $n m^{n}$ defined as

$$
A \curlywedge B=I_{m}^{\otimes^{n}} \otimes A+\sum_{i=1}^{n} I_{m}^{\otimes^{i-1}} \otimes B \otimes I_{m}^{\otimes^{n-i}} \otimes C_{i} .
$$

In [8] the following theorem, which shows the correspondence between wreath products of matrices and wreath products of graphs, is proven.

Theorem 2.3. Let $A_{1}^{\prime}$ be the normalized adjacency matrix of a $d_{1}$-regular graph $\mathcal{G}_{1}=$ $\left(V_{1}, E_{1}\right)$ and let $A_{2}^{\prime}$ be the normalized adjacency matrix of a $d_{2}$-regular graph $\mathcal{G}_{2}=$ $\left(V_{2}, E_{2}\right)$. Then the wreath product $\left(\frac{d_{1}}{d_{1}+d_{2}} A_{1}^{\prime}\right)$ ᄀ $\left(\frac{d_{2}}{d_{1}+d_{2}} A_{2}^{\prime}\right)$ is the normalized adjacency matrix of the graph wreath product $\mathcal{G}_{1} \prec \mathcal{G}_{2}$.

For a finite connected graph $\mathcal{G}=(V, E)$, the distance matrix $D=\left(d_{u, v}\right)_{u, v \in V}$ of $\mathcal{G}$ is, by definition, the square matrix indexed by the vertices of $\mathcal{G}$, such that $d_{u, v}=d(u, v)$. The matrix $D$ is symmetric by definition, so that its spectrum is real. The spectrum of $D$ is usually called the distance spectrum of the graph $\mathcal{G}$.

We complete this preliminary section by recalling the definition of Wiener index of a finite connected graph $\mathcal{G}=(V, E)$. The Wiener index of $\mathcal{G}$ is defined as the sum of the distances between all the unordered pairs of vertices, i.e.,

$$
W(\mathcal{G})=\frac{1}{2} \sum_{u, v \in V} d(u, v)
$$


where $d(u, v)$ denotes the usual geodesic distance between $u$ and $v$.

In Section 3, we will construct the adjacency matrix and the distance matrix of the graph $K_{n} \prec K_{m}$, and we will compute their spectra. Finally, we will provide an explicit computation of the Wiener index of $K_{n} \prec K_{m}$.

\section{The wreath product $K_{n} \succ K_{m}$}

From now on, we will focus our attention on the wreath product $K_{n} \prec K_{m}$, where $K_{n}=$ $\left(V_{n}, E_{n}\right)$ is the complete graph on $n$ vertices, that is, the graph on $n$ vertices in which every pair of distinct vertices is connected by a unique edge. Notice that $K_{n}$ is a regular graph of degree $n-1$, with diameter 1 , where $d(u, v)=\left\{\begin{array}{ll}0 & \text { if } u=v \\ 1 & \text { if } u \neq v\end{array}\right.$ for each pair $u, v$ of vertices.

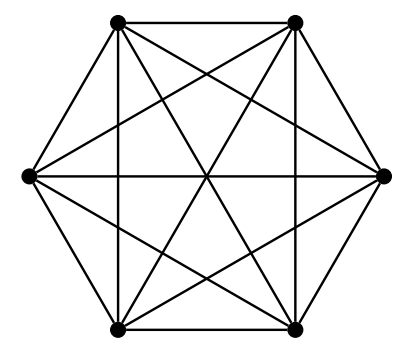

Figure 1: The complete graph $K_{6}$.

In particular, the adjacency matrix of $K_{n}$ is given by $A d_{n}=J_{n}-I_{n}$, where $J_{n}$ denotes the uniform square matrix of size $n$, whose entries are all equal to 1 . Moreover, it follows from Theorem 2.3 that the adjacency matrix of the graph $K_{n} \prec K_{m}$ is the matrix

$$
A d_{n} \curlywedge A d_{m}=I_{m}^{\otimes^{n}} \otimes A d_{n}+\sum_{i=1}^{n} I_{m}^{\otimes^{i-1}} \otimes A d_{m} \otimes I_{m}^{\otimes^{n-i}} \otimes C_{i},
$$

with $C_{i}$ as in Definition 2.2. Notice also that, by definition, $K_{n} \prec K_{m}$ is an $(n+m-$ 2)-regular graph on $n m^{n}$ vertices. A vertex of $K_{n} \prec K_{m}$ will be usually denoted by $\left(y_{1}, \ldots, y_{n}\right) x_{i}$, where $y_{j} \in V_{m}$, for each $j=1, \ldots, n$, and $x_{i} \in V_{n}$. In the lamplighter interpretation, we can think that the lamp placed at the $j$-th vertex $x_{j}$ of $K_{n}$ has color $y_{j}$, with $y_{j} \in V_{m}$, and the lamplighter is in position $x_{i}$.

Moreover, it follows from the definition of wreath product of graphs that two vertices $u=\left(y_{1}, \ldots, y_{n}\right) x_{i}$ and $v=\left(y_{1}^{\prime}, \ldots, y_{n}^{\prime}\right) x_{k}$ have distance 1 if either there exists a unique index $j \in\{1, \ldots, n\}$ such that $y_{j} \neq y_{j}^{\prime}$ and $x_{i}=x_{k}$; or $y_{j}=y_{j}^{\prime}$ for each $j$, and $x_{i} \neq x_{k}$ (observe that $x_{i} \sim x_{k}$ in $K_{n}$ if and only if $x_{i} \neq x_{k}$, as the graph $K_{n}$ is complete).

We are going to develop an explicit analysis of the variability of the distances between two vertices in the graph $K_{n} \imath K_{m}$. Let $u=\left(y_{1}, \ldots, y_{n}\right) x_{i}$ and $v=\left(y_{1}^{\prime}, \ldots, y_{n}^{\prime}\right) x_{k}$ be two vertices of $K_{n} \prec K_{m}$. Put $J=\{1,2, \ldots, n\}$ and define the partition $J=J_{u, v}^{0} \sqcup J_{u, v}^{1}$ by

$$
J_{u, v}^{0}=\left\{j \in J: y_{j}=y_{j}^{\prime}\right\} \quad J_{u, v}^{1}=\left\{j \in J: y_{j} \neq y_{j}^{\prime}\right\} .
$$

Note that the cardinality $\left|J_{u, v}^{1}\right|$ can be interpreted as the Hamming distance between the "lamp strings" $\left(y_{1}, \ldots, y_{n}\right)$ and $\left(y_{1}^{\prime}, \ldots, y_{n}^{\prime}\right)$. The following proposition holds. 
Proposition 3.1. Let $u=\left(y_{1}, \ldots, y_{n}\right) x_{i}$ and $v=\left(y_{1}^{\prime}, \ldots, y_{n}^{\prime}\right) x_{k}$ be two vertices of $K_{n}$ ? $K_{m}$ and let $J_{u, v}^{0}$ and $J_{u, v}^{1}$ as in (3.2). Then

$$
\begin{aligned}
& d(u, v)=\left\{\begin{array}{ll}
0 & \text { if } i=k \\
1 & \text { if } i \neq k
\end{array} \quad \text { if } J_{u, v}^{1}=\emptyset ;\right. \\
& d(u, v)=\left\{\begin{array}{ll}
1 & \text { if } i=k=j_{*} \\
3 & \text { if } i \neq j_{*} \neq k \\
2 & \text { if } i=j_{*} \neq k ; \text { or } i \neq j_{*}=k
\end{array} \quad \text { if } J_{u, v}^{1}=\left\{j_{*}\right\} .\right.
\end{aligned}
$$

More generally, for $2 \leq\left|J_{u, v}^{1}\right| \leq n$ :

$$
d(u, v)= \begin{cases}2\left|J_{u, v}^{1}\right|+1 & \text { if } k, i \in J_{u, v}^{0} \\ 2\left|J_{u, v}^{1}\right| & \text { if } i \in J_{u, v}^{0}, k \in J_{u, v}^{1} ; \text { or } i \in J_{u, v}^{1}, k \in J_{u, v}^{0} \\ 2\left|J_{u, v}^{1}\right|-1+\delta_{i k} & \text { if } i, k \in J_{u, v}^{1}\end{cases}
$$

$$
\text { with } \delta_{i k}= \begin{cases}1 & \text { if } i=k \\ 0 & \text { if } i \neq k\end{cases}
$$

Proof. First of all observe that, if $J_{u, v}^{1}=\emptyset$, we have $y_{j}=y_{j}^{\prime}$ for each $j \in J$, so that $u$ and $v$ coincide if $i=k$, whereas they are adjacent, by an edge of type II in $K_{n} \imath K_{m}$, if $i \neq k$.

Suppose now $J_{u, v}^{1}=\left\{j_{*}\right\}$. In the first case, the vertices $u=\left(y_{1}, \ldots, y_{j_{*}}, \ldots, y_{n}\right) x_{j_{*}}$ and $v=\left(y_{1}, \ldots, y_{j_{*}}^{\prime}, \ldots, y_{n}\right) x_{j_{*}}$, with $y_{j_{*}} \neq y_{j_{*}}^{\prime}$, are adjacent in $K_{n} \imath K_{m}$. In the second case, when $i \neq j_{*} \neq k$, the path

$$
\begin{array}{r}
\left(y_{1}, \ldots, y_{j_{*}}, \ldots, y_{n}\right) x_{i} \sim\left(y_{1}, \ldots, y_{j_{*}}, \ldots, y_{n}\right) x_{j_{*}} \sim\left(y_{1}, \ldots, y_{j_{*}}^{\prime}, \ldots, y_{n}\right) x_{j_{*}} \sim \\
\\
\sim\left(y_{1}, \ldots, y_{j_{*}}^{\prime}, \ldots, y_{n}\right) x_{k}
\end{array}
$$

is a path of minimal length joining $u$ and $v$. In the third case, when $i=j_{*} \neq k$, the path

$$
\left(y_{1}, \ldots, y_{j_{*}}, \ldots, y_{n}\right) x_{j_{*}} \sim\left(y_{1}, \ldots, y_{j_{*}}^{\prime}, \ldots, y_{n}\right) x_{j_{*}} \sim\left(y_{1}, \ldots, y_{j_{*}}^{\prime}, \ldots, y_{n}\right) x_{k}
$$

is a path of minimal length joining $u$ and $v$; the case $i \neq j_{*}=k$ is similar.

Now let $2 \leq\left|J_{u, v}^{1}\right|=h \leq n$, with $J_{u, v}^{1}=\left\{j_{1}, \ldots, j_{h}\right\}$. In other words, the $n$-tuples $\left(y_{1}, \ldots, y_{n}\right)$ and $\left(y_{1}^{\prime}, \ldots, y_{n}^{\prime}\right)$ differ exactly in $h$ places, indexed by the elements $j_{1}, \ldots, j_{h}$ of $J_{u, v}^{1}$. In the first case, the path

$$
\begin{gathered}
\left(y_{1}, \ldots, y_{j_{1}}, \ldots, y_{n}\right) x_{i} \sim\left(y_{1}, \ldots, y_{j_{1}}, \ldots, y_{n}\right) x_{j_{1}} \sim\left(y_{1}, \ldots, y_{j_{1}}^{\prime}, \ldots, y_{n}\right) x_{j_{1}} \sim \\
\sim\left(y_{1}, \ldots, y_{j_{1}}^{\prime}, \ldots, y_{n}\right) x_{j_{2}} \sim \ldots \sim\left(y_{1}, \ldots, y_{j_{1}}^{\prime}, \ldots, y_{j_{h-1}}^{\prime}, \ldots, y_{n}\right) x_{j_{h}} \sim \\
\sim\left(y_{1}, \ldots, y_{j_{1}}^{\prime}, \ldots, y_{j_{h-1}}^{\prime}, \ldots, y_{j_{h}}^{\prime}, \ldots, y_{n}\right) x_{j_{h}} \sim \\
\sim\left(y_{1}, \ldots, y_{j_{1}}^{\prime}, \ldots, y_{j_{h-1}}^{\prime}, \ldots, y_{j_{h}}^{\prime}, \ldots, y_{n}\right) x_{k}
\end{gathered}
$$

is a minimal path joining $u$ and $v$, and it has length $2 h+1$. In the second case, when $i \in J_{u, v}^{0}, k \in J_{u, v}^{1}$, we can assume, without loss of generality, because $K_{n}$ is complete, that $j_{h}=k$, so that the last step is not necessary, and a path of minimal length connecting $u$ and $v$ has length $2 h$; a similar argument works in the case $i \in J_{u, v}^{1}, k \in J_{u, v}^{0}$. Finally, if $i \neq k$ and $i, k \in J_{u, v}^{1}$, we can assume that $j_{1}=i$ and $j_{h}=k$. Now, a path of minimal 
length joining $u$ and $v$ is given by

$$
\begin{aligned}
\left(y_{1}, \ldots, y_{j_{1}}, \ldots, y_{n}\right) & x_{j_{1}} \sim\left(y_{1}, \ldots, y_{j_{1}}^{\prime}, \ldots, y_{n}\right) x_{j_{1}} \sim\left(y_{1}, \ldots, y_{j_{1}}^{\prime}, \ldots, y_{n}\right) x_{j_{2}} \sim \\
& \sim\left(y_{1}, \ldots, y_{j_{1}}^{\prime}, \ldots, y_{j_{2}}^{\prime}, \ldots, y_{n}\right) x_{j_{2}} \sim \ldots \sim \\
& \sim\left(y_{1}, \ldots, y_{j_{1}}^{\prime}, \ldots, y_{j_{2}}^{\prime} \ldots, y_{j_{h-1}}^{\prime}, \ldots, y_{n}\right) x_{j_{h-1}} \sim \\
& \sim\left(y_{1}, \ldots, y_{j_{1}}^{\prime}, \ldots, y_{j_{2}}^{\prime} \ldots, y_{j_{h-1}}^{\prime}, \ldots, y_{n}\right) x_{j_{h}} \sim \\
& \sim\left(y_{1}, \ldots, y_{j_{1}}^{\prime}, \ldots, y_{j_{h-1}}^{\prime}, \ldots, y_{j_{h}}^{\prime}, \ldots, y_{n}\right) x_{j_{h}}
\end{aligned}
$$

and has length $2 h-1$. In the special case $i=k$, we need one more step in order to reach the final vertex $x_{k}=x_{i}$, by means of an edge of type II in $K_{n} \imath K_{m}$.

Corollary 3.2. The diameter of the graph $K_{n} \prec K_{m}$ is $2 n$.

Proof. It follows from the proof of Proposition 3.1 that the maximal distance $d(u, v)$ between two vertices $u$ and $v$ of $K_{n} \imath K_{m}$ is equal to $2 n$, and it is obtained when the vertices $u, v$ have the form

$$
u=\left(y_{1}, \ldots, y_{n}\right) x_{i} \quad v=\left(y_{1}^{\prime}, \ldots, y_{n}^{\prime}\right) x_{k},
$$

with $y_{j} \neq y_{j}^{\prime}$, for each $j=1, \ldots, n$ and $x_{i}=x_{k}$. In fact, we get in this case

$$
d(u, v)=2\left|J_{u, v}^{1}\right|-1+\delta_{i k}=2 n-1+1=2 n .
$$

Now, for each $i=0,1, \ldots, 2 n$, and every vertex $u$ of $K_{n} \prec K_{m}$, we denote by $S_{i}(u)$ the sphere of radius $i$ centered at $u$, that is:

$$
S_{i}(u)=\left\{v \in V\left(K_{n} \curlywedge K_{m}\right): d(u, v)=i\right\} .
$$

Because of the complete symmetry of the graph, it is clear that the integer $s_{i}=\left|S_{i}(u)\right|$ does not depend on the particular choice of the vertex $u$. We recall below the classical definition of distance-regular graph (see, for instance, [4], or [5, Chapter 12] for some results about spectral properties of distance-regular graphs, also in connection with association scheme theory).

Definition 3.3. A connected graph $\mathcal{G}$ is said to be distance-regular if it is regular and, for any two vertices $u, v$ at distance $i$, there are exactly $c_{i}$ neighbors of $v$ in $S_{i-1}(u)$ and $b_{i}$ neighbors of $v$ in $S_{i+1}(u)$.

If $d$ is the diameter of $\mathcal{G}$, the sequence $\left\{b_{0}, b_{1}, \ldots, b_{d-1} ; c_{1}, c_{2}, \ldots, c_{d}\right\}$ is usually called the intersection array of $\mathcal{G}$; notice that the integers $c_{0}$ and $b_{d}$ are undefined.

Proposition 3.4. For every $n, m$, the wreath product $K_{n} \curlywedge K_{m}$ is not distance-regular.

Proof. Consider two vertices of type $u=\left(y_{1}, \ldots, y_{n}\right) x_{i}$ and $v=\left(y_{1}, \ldots, y_{n}\right) x_{j}$, with $j \neq i$, so that $d(u, v)=1$. Now, the neighbors of $v$ having distance 2 from $u$ are exactly the vertices of type $\left(y_{1}, \ldots, y_{j}^{\prime}, \ldots, y_{n}\right) x_{j}$, with $y_{j}^{\prime} \neq y_{j}$ : the number of such vertices is $m-1$. On the other hand, consider the vertex $w=\left(y_{1}, \ldots, y_{i}^{\prime}, \ldots, y_{n}\right) x_{i}$, with $y_{i}^{\prime} \neq y_{i}$, so that we still have $d(u, w)=1$. It is clear that the neighbors of $w$ having distance 2 
from $u$ are exactly the vertices of type $\left(y_{1}, \ldots, y_{i}^{\prime}, \ldots, y_{n}\right) x_{j}$, with $x_{j} \neq x_{i}$, and they are precisely $n-1$. This implies that the coefficient $b_{1}$ cannot be defined, and this is sufficient to conclude that $K_{n} \imath K_{m}$ is not distance-regular.

Also in the case $n=m$, the graph is not distance-regular. In order to show that, it suffices to consider the vertices $u=\left(y_{1}, \ldots, y_{n-1}, y_{n}\right) x_{n}$ and $v=\left(y_{1}^{\prime}, \ldots, y_{n-1}^{\prime}, y_{n}\right) x_{n}$, with $y_{j}^{\prime} \neq y_{j}$ for each $j=1, \ldots, n-1$, so that $d(u, v)=2 n-1$, according to Proposition 3.1. Now, the neighbors of $v$ having distance $2 n$ from $u$ are exactly the vertices of type $\left(y_{1}^{\prime}, \ldots, y_{n-1}^{\prime}, y_{n}^{\prime}\right) x_{n}$, with $y_{n}^{\prime} \neq y_{n}$ : the number of such vertices is $n-1$.

On the other hand, consider the vertices $w=\left(y_{1}, \ldots, y_{n}\right) x_{i}$ and $z=\left(y_{1}^{\prime}, \ldots, y_{n}^{\prime}\right) x_{j}$, with $y_{j}^{\prime} \neq y_{j}$ for each $j=1, \ldots, n$ and $x_{i} \neq x_{j}$, so that we still have $d(w, z)=2 n-1$. In this case, the unique neighbor of $z$ having distance $2 n$ from $w$ is the vertex $\left(y_{1}^{\prime}, \ldots, y_{n}^{\prime}\right) x_{i}$. This implies that the coefficient $b_{2 n-1}$ cannot be defined, and this is sufficient to conclude that $K_{n} \prec K_{n}$ is not distance-regular.

Example 3.5. Consider the graph $K_{3}<K_{2}$ depicted in Figure 2, where the vertices of $K_{3}$ and $K_{2}$ are identified with the sets $\{0,1,2\}$ and $\{0,1\}$, respectively. The adjacency matrices of the graphs $K_{3}$ and $K_{2}$ are, respectively,

$$
A d_{3}=\left(\begin{array}{ccc}
0 & 1 & 1 \\
1 & 0 & 1 \\
1 & 1 & 0
\end{array}\right) \quad \text { and } \quad A d_{2}=\left(\begin{array}{cc}
0 & 1 \\
1 & 0
\end{array}\right)
$$

so that the matrix wreath product

$$
\begin{aligned}
A d_{3} \zeta A d_{2}=I_{2}^{\otimes^{3}} \otimes A d_{3}+A d_{2} \otimes I_{2} & \otimes I_{2} \otimes C_{1}+ \\
& +I_{2} \otimes A d_{2} \otimes I_{2} \otimes C_{2}+I_{2} \otimes I_{2} \otimes A d_{2} \otimes C_{3}
\end{aligned}
$$

is the adjacency matrix of the graph $K_{3} \imath K_{2}$. The graph $K_{3} \imath K_{2}$ is regular of degree 3 , and its diameter is 6 .

\subsection{Spectrum of the graph $K_{n} \succ K_{m}$}

In this section, we will give an explicit description of the spectrum of the graph $K_{n}$ ? $K_{m}$ which is, by definition, the spectrum of its adjacency matrix $A d_{n} \prec A d_{m}$ described in Equation (3.1). In order to develop our analysis, we need to recall the definition of circulant matrix. A (complex) circulant matrix $C$ of size $m$ is a square matrix with $m$ rows and $m$ columns, of type

$$
C=\left(\begin{array}{ccccc}
c_{0} & c_{1} & \cdots & \cdots & c_{m-1} \\
c_{m-1} & c_{0} & c_{1} & & \vdots \\
\vdots & \ddots & \ddots & \ddots & \vdots \\
\vdots & & \ddots & \ddots & c_{1} \\
c_{1} & \cdots & \cdots & c_{m-1} & c_{0}
\end{array}\right) \quad \text { with } c_{i} \in \mathbb{C}, \forall i=0, \ldots, m-1
$$

The reader can refer to [11] as an exhaustive monograph on circulant matrices.

The following theorem has been proven in [8], by using the spectral analysis developed in [35] for block circulant matrices. 


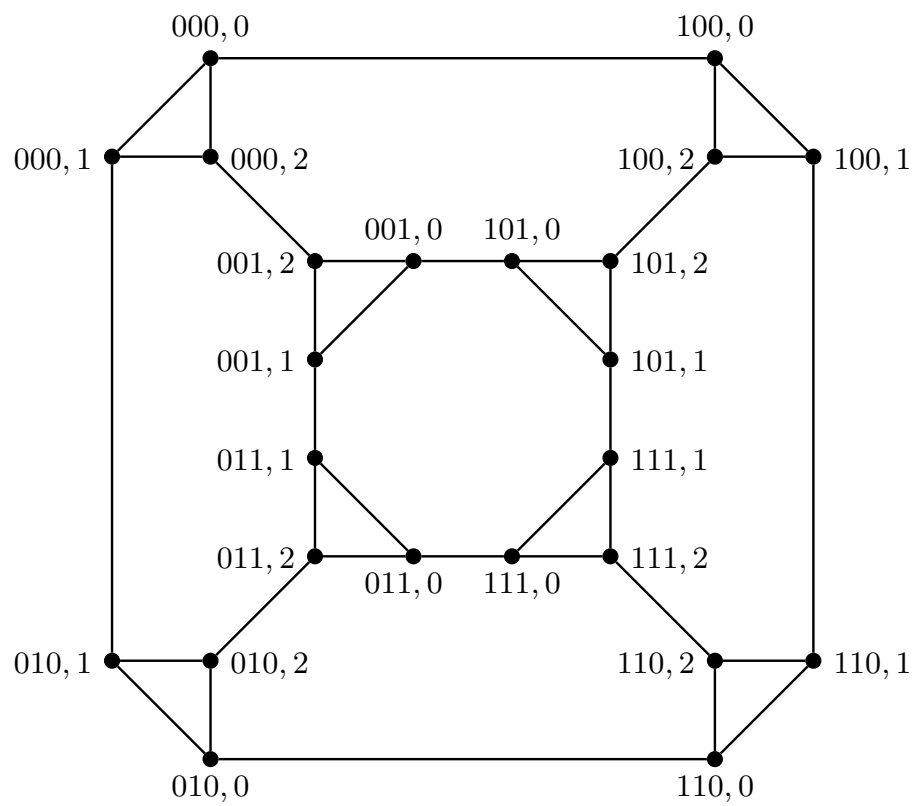

Figure 2: The graph $K_{3} \succ K_{2}$.

Theorem 3.6. Let $A$ be a square matrix of size $n$, and let $B$ be a circulant matrix of size $m$ as in (3.3). Then the spectrum $\Sigma$ of the matrix $A$ < $B$ is obtained by taking the union of the spectra $\Sigma_{i_{1}, \ldots, i_{n}}$ of the $m^{n}$ matrices of size $n$ given by

$$
\widetilde{M}^{i_{1}, i_{2}, \ldots, i_{n}}=A+\sum_{t=1}^{n} \sum_{i=0}^{m-1} c_{i} \rho^{i i_{t}} C_{t},
$$

where $i_{j} \in\{0,1, \ldots, m-1\}$, for every $j=1, \ldots, n$, and $\rho=\exp \left(\frac{2 \pi i}{m}\right)$.

In particular, Theorem 3.6 can be applied in order to determine the spectrum of the adjacency matrix

$$
A d_{n} \curlywedge A d_{m}=I_{m}^{\otimes^{n}} \otimes A d_{n}+\sum_{i=1}^{n} I_{m}^{\otimes^{i-1}} \otimes A d_{m} \otimes I_{m}^{\otimes^{n-i}} \otimes C_{i},
$$

since the matrix $A d_{m}$ is a circulant matrix, with $c_{0}=0$ and $c_{i}=1$, for each $i=1, \ldots, m-$ 1. When listing eigenvalues and their multiplicities in the next theorem, and in the rest of the paper, we will write $\lambda^{h}$ to say that the eigenvalue $\lambda$ has multiplicity $h$; the multiplicity will be omitted when it is equal to 1 . We obtain the following result.

Theorem 3.7. The spectrum $\Sigma$ of the graph $K_{n} \curlywedge K_{m}$ is $\Sigma=\bigcup_{k=0}^{n} \Sigma_{k}^{\left(\begin{array}{l}n \\ k\end{array}\right) \cdot(m-1)^{n-k}}$, with

$$
\begin{aligned}
& \Sigma_{0}=\left\{(-2)^{n-1} ; n-2\right\} \\
& \Sigma_{k}=\left\{(m-2)^{k-1} ;(-2)^{n-k-1} ; \frac{m+n-4 \pm \sqrt{(m-n)^{2}+4 k m}}{2}\right\}, k=1, \ldots, n-1
\end{aligned}
$$


and

$$
\Sigma_{n}=\left\{(m-2)^{n-1} ; m+n-2\right\} .
$$

Proof. By virtue of Theorem 3.6, the spectrum of $K_{n} \prec K_{m}$ is obtained by taking the union of the spectra $\Sigma_{i_{1}, \ldots, i_{n}}$ of the matrices

$$
\widetilde{M}^{i_{1}, i_{2}, \ldots, i_{n}}=A d_{n}+\sum_{t=1}^{n} \sum_{i=0}^{m-1} c_{i} \rho^{i i_{t}} C_{t},
$$

where $i_{j} \in\{0,1, \ldots, m-1\}$, for each $j=1, \ldots, n$, and $\rho=\exp \left(\frac{2 \pi i}{m}\right)$. Notice that $c_{0}=0$ and $c_{i}=1$ for each $i=1, \ldots, m-1$. Moreover, the following identity holds:

$$
\sum_{i=1}^{m-1}\left(\rho^{i_{t}}\right)^{i}= \begin{cases}m-1 & \text { if } i_{t}=0 \\ \frac{\left(\rho^{i_{t}}\right)^{m}-1}{\rho^{i_{t}}-1}-1=-1 & \text { if } i_{t} \neq 0\end{cases}
$$

since $\rho$ is an $m$-th root of unity. Therefore, the matrix $\widetilde{M}^{i_{1}, i_{2}, \ldots, i_{n}}$ can be rewritten as

$$
\begin{aligned}
\widetilde{M}^{i_{1}, i_{2}, \ldots, i_{n}} & =A d_{n}+\sum_{t: i_{t}=0}(m-1) C_{t}-\sum_{t: i_{t} \neq 0} C_{t} \\
& =J_{n}-I_{n}+\sum_{t: i_{t}=0}(m-1) C_{t}-\left(\sum_{t: i_{t} \neq 0} C_{t}+\sum_{t: i_{t}=0} C_{t}\right)+\sum_{t: i_{t}=0} C_{t} \\
& =J_{n}-2 I_{n}+m \sum_{t: i_{t}=0} C_{t} .
\end{aligned}
$$

By using iterated conjugations with appropriate elementary permutation matrices, it can be shown that the spectrum of the matrix $\widetilde{M}^{i_{1}, i_{2}, \ldots, i_{n}}$ only depends on the number $k$ of indices equal to 0 in the $n$-tuple $\left(i_{1}, i_{2}, \ldots, i_{n}\right)$, but it is independent of the particular position of such indices. As a consequence, for each $k=0,1, \ldots, n$, we can reduce to investigate the spectrum of the matrix $\widetilde{M}^{0, \ldots, 0, i_{k+1}, \ldots, i_{n}}$, corresponding to the $n$-tuple $(\underbrace{0, \ldots, 0}_{k \text { times }}, i_{k+1}, \ldots, i_{n})$, with $i_{j} \neq 0$ for each $j=k+1, \ldots, n$. We have:

$$
\widetilde{M}^{0, \ldots, 0, i_{k+1}, \ldots, i_{n}}=\left(\begin{array}{cccccc}
-1+m & 1 & \cdots & \cdots & \cdots & 1 \\
1 & \ddots & 1 & & & \vdots \\
1 & 1 & -1+m & 1 & \cdots & \vdots \\
\vdots & & & -1 & & 1 \\
\vdots & & & 1 & \ddots & \vdots \\
1 & \cdots & \cdots & \cdots & 1 & -1
\end{array}\right) .
$$

Then we can write $\widetilde{M}^{0, \ldots, 0, i_{k+1}, \ldots, i_{n}}=J_{n}+Q$, where $Q=m \sum_{t=1}^{k} C_{t}-2 I_{n}$ is the 
diagonal matrix

$$
Q=\left(\begin{array}{cccccc}
-2+m & & & & & \\
& \ddots & & & & \\
& & -2+m & & & \\
& & & -2 & & \\
& & & & \ddots & \\
& & & & & -2
\end{array}\right)
$$

Now we have:

$$
\begin{aligned}
\operatorname{det}\left(\lambda I_{n}-\widetilde{M}^{0, \ldots, 0, i_{k+1}, \ldots, i_{n}}\right) & =\operatorname{det}\left(\lambda I_{n}-J_{n}-Q\right) \\
& =\operatorname{det}\left(\left(\lambda I_{n}-Q\right)\left(I_{n}-\left(\lambda I_{n}-Q\right)^{-1} J_{n}\right)\right) \\
& =\operatorname{det}\left(\lambda I_{n}-Q\right) \cdot \operatorname{det}\left(I_{n}-\left(\lambda I_{n}-Q\right)^{-1} J_{n}\right) .
\end{aligned}
$$

It is clear that

$$
\operatorname{det}\left(\lambda I_{n}-Q\right)=(\lambda-(m-2))^{k} \cdot(\lambda+2)^{n-k} .
$$

Now it can be seen that the matrix $\left(\lambda I_{n}-Q\right)^{-1} J_{n}$ is the matrix of rank 1 , whose first $k$ rows are constant, with entries all equal to $\frac{1}{\lambda-(m-2)}$, whereas the remaining $n-k$ rows are constant, with entries all equal to $\frac{1}{\lambda+2}$. Therefore, $\left(\lambda I_{n}-Q\right)^{-1} J_{n}$ has $n-1$ eigenvalues equal to 0 , and one eigenvalue equal to $\frac{k}{\lambda-(m-2)}+\frac{n-k}{\lambda+2}$. This implies that the matrix $I_{n}-\left(\lambda I_{n}-Q\right)^{-1} J_{n}$ has $n-1$ eigenvalues equal to 1 , and one eigenvalue equal to $1-$ $\frac{k}{\lambda-(m-2)}-\frac{n-k}{\lambda+2}$, so that:

$$
\operatorname{det}\left(I_{n}-\left(\lambda I_{n}-Q\right)^{-1} J_{n}\right)=1-\frac{k}{\lambda-(m-2)}-\frac{n-k}{\lambda+2} .
$$

By gluing together (3.4) and (3.5), we obtain:

$$
\begin{aligned}
\operatorname{det}\left(\lambda I_{n}-\widetilde{M}^{0, \ldots, 0, i_{k+1}, \ldots, i_{n}}\right) & =(\lambda-(m-2))^{k-1} \cdot(\lambda+2)^{n-k-1} . \\
& \cdot\left(\lambda^{2}+(4-m-n) \lambda+m n+4-k m-2 n-2 m\right) .
\end{aligned}
$$

For the particular value $k=0$, we get:

$$
\operatorname{det}\left(\lambda I_{n}-\widetilde{M}^{i_{1}, \ldots, i_{n}}\right)=(\lambda+2)^{n-1} \cdot(\lambda-(n-2))
$$

for the particular value $k=n$, we have:

$$
\operatorname{det}\left(\lambda I_{n}-\widetilde{M}^{0, \ldots, 0}\right)=(\lambda-(m-2))^{n-1} \cdot(\lambda-(m+n-2)) .
$$

The claim follows, if we observe that, for each $k=0,1, \ldots, n$, the spectrum of $\Sigma_{k}$ must be considered $\left(\begin{array}{l}n \\ k\end{array}\right) \cdot(m-1)^{n-k}$ times, corresponding to the number of $n$-tuples $\left(i_{1}, \ldots, i_{n}\right)$ with $k$ indices equal to 0 , and the remaining indices varying in $\{1, \ldots, m-1\}$. 
Example 3.8. Consider the graph $K_{3} \prec K_{4}$, so that $n=3$ and $m=4$. The spectrum of the matrix $A d_{3}<A d_{4}$ consists of the following eigenvalues:

$$
5 ; 2^{11} ; 1^{27} ;(-2)^{81} ;\left(\frac{3 \pm \sqrt{17}}{2}\right)^{27} ;\left(\frac{3 \pm \sqrt{33}}{2}\right)^{9} .
$$

The corresponding matrices $\widetilde{M}^{i_{1}, i_{2}, i_{3}}$ of size 3 , with $i_{1}, i_{2}, i_{3} \in\{0,1,2,3\}$, have eigenvalues:

(a) $(-2)^{2} ; 1$, for $k=0$. For instance, this is the case of the matrix

$$
\widetilde{M}^{1,1,1}=J_{3}-2 I_{3}=\left(\begin{array}{ccc}
-1 & 1 & 1 \\
1 & -1 & 1 \\
1 & 1 & -1
\end{array}\right)
$$

(b) $-2 ; \frac{3 \pm \sqrt{17}}{2}$, for $k=1$. For instance, this is the case of the matrix

$$
\widetilde{M}^{0,1,1}=J_{3}-2 I_{3}+4 C_{1}=\left(\begin{array}{ccc}
3 & 1 & 1 \\
1 & -1 & 1 \\
1 & 1 & -1
\end{array}\right)
$$

(c) $2 ; \frac{3 \pm \sqrt{33}}{2}$, for $k=2$. For instance, this is the case of the matrix

$$
\widetilde{M}^{0,0,1}=J_{3}-2 I_{3}+4\left(C_{1}+C_{2}\right)=\left(\begin{array}{ccc}
3 & 1 & 1 \\
1 & 3 & 1 \\
1 & 1 & -1
\end{array}\right) .
$$

(d) $2^{2} ; 5$, for $k=3$. This is the case of the matrix

$$
\widetilde{M}^{0,0,0}=J_{3}-2 I_{3}+4\left(C_{1}+C_{2}+C_{3}\right)=J_{3}+2 I_{3}=\left(\begin{array}{ccc}
3 & 1 & 1 \\
1 & 3 & 1 \\
1 & 1 & 3
\end{array}\right) .
$$

Corollary 3.9. The spectrum $\Sigma^{\prime}$ of the transition matrix of the Lamplighter random walk with base graph $K_{n}$ and color graph $K_{m}$ is $\Sigma^{\prime}=\bigcup_{k=0}^{n} \Sigma_{k}^{\prime}\left(\begin{array}{l}n \\ k\end{array}\right) \cdot(m-1)^{n-k}$, with

$$
\begin{aligned}
& \Sigma_{0}^{\prime}=\left\{\left(-\frac{2}{m+n-2}\right)^{n-1} ; \frac{n-2}{m+n-2}\right\} \\
& \Sigma_{k}^{\prime}=\left\{\left(\frac{m-2}{m+n-2}\right)^{k-1} ;\left(-\frac{2}{m+n-2}\right)^{n-k-1} ; \frac{m+n-4 \pm \sqrt{(m-n)^{2}+4 k m}}{2(m+n-2)}\right\},
\end{aligned}
$$

for $k=1, \ldots, n-1$, and

$$
\Sigma_{n}^{\prime}=\left\{\left(\frac{m-2}{m+n-2}\right)^{n-1} ; 1\right\} \text {. }
$$

Proof. It suffices to take into account that the transition matrix of the Lamplighter random walk on the base graph $K_{n}$, with color graph $K_{m}$, is the normalized adjacency matrix of the graph $K_{n} \prec K_{m}$, which is a regular graph of degree $m+n-2$. 


\subsection{Distance spectrum and Wiener index of the graph $K_{n} \succ K_{m}$}

The aim of this section is to describe the distance matrix of the graph $K_{n} \prec K_{m}$, together with its spectrum. Moreover, we will exhibit an explicit computation of the Wiener index of the graph.

Proposition 3.10. The distance matrix of the graph $K_{n} \prec K_{m}$ is the matrix

$$
D=\sum_{\left(i_{1}, \ldots, i_{n}\right) \in\{0,1\}^{n}} A d_{m}^{i_{1}} \otimes A d_{m}^{i_{2}} \otimes \cdots \otimes A d_{m}^{i_{n}} \otimes A_{i_{1}, i_{2}, \ldots, i_{n}},
$$

where we put $A d_{m}^{0}=I_{m}$, and the matrix $A_{i_{1}, i_{2}, \ldots, i_{n}}$ is the square matrix of size $n$, indexed by the vertices of $K_{n}$, defined as follows. Let $\left\{i_{1}, \ldots, i_{n}\right\}=I_{0} \sqcup I_{1}$, with $I_{0}=\left\{i_{j}: i_{j}=\right.$ $0\}$ and $I_{1}=\left\{i_{j}: i_{j}=1\right\}$. Then, for any pair of vertices $x_{i}$ and $x_{k}$ of $K_{n}$ :

(a) $A_{i_{1}, \ldots, i_{n}}=A d_{n}=J_{n}-I_{n}$ if $I_{1}=\emptyset$;

(b) $A_{i_{1}, \ldots, i_{n}}\left(x_{i}, x_{k}\right)=\left\{\begin{array}{ll}1 & \text { if } i=k=j_{*} \\ 3 & \text { if } i \neq j_{*} \neq k \\ 2 & \text { if } i=j_{*} \neq k ; \text { or } i \neq j_{*}=k\end{array} \quad\right.$ if $I_{1}=\left\{i_{j_{*}}\right\}$;

(c) $A_{i_{1}, \ldots, i_{n}}\left(x_{i}, x_{k}\right)= \begin{cases}2\left|I_{1}\right|+1 & \text { if } i, k \in I_{0} \\ 2\left|I_{1}\right| & \text { if } i \in I_{0}, k \in I_{1} ; \text { or } i \in I_{1}, k \in I_{0} \\ 2\left|I_{1}\right|-1+\delta_{i k} & \text { if } i, k \in I_{1}\end{cases}$

$$
\text { if } 2 \leq\left|I_{1}\right| \leq n \text {, where } \delta_{i k}=\left\{\begin{array}{ll}
1 & \text { if } i=k \\
0 & \text { if } i \neq k
\end{array} .\right.
$$

Proof. Observe that, for each $j=1, \ldots, n$, the index $i_{j} \in\{0,1\}$ establishes whether the color of the lamp at the $j$-th vertex $x_{j}$ of $K_{n}$ is changed. More precisely, the index $i_{j}=0$ produces the matrix $A d_{m}^{0}=I_{m}$ as $j$-th term of the Kronecker product, so that we are not changing the color of the lamp in that position; conversely, the index $i_{j}=1$ provides the matrix $A d_{m}$ as $j$-th term of the Kronecker product, so that we are changing the color of the lamp in that position, with any other color, as $K_{m}$ is the complete graph. Therefore, for any fixed $n$-tuple $\left(i_{1}, \ldots, i_{n}\right) \in\{0,1\}^{n}$, the contribution $A d_{m}^{i_{1}} \otimes A d_{m}^{i_{2}} \otimes \cdots \otimes A d_{m}^{i_{n}} \otimes A_{i_{1}, i_{2}, \ldots, i_{n}}$ to $D$ must take into account the distances between vertices $u, v$ of $K_{n} \imath K_{m}$ corresponding to lamp configurations which differ exactly at the places indexed by $I_{1}$. Therefore, if the configurations of lamps corresponding to the vertices $u=\left(y_{1}, \ldots, y_{n}\right) x_{i}$ and $v=$ $\left(y_{1}^{\prime}, \ldots, y_{n}^{\prime}\right) x_{k}$ of $K_{n} \prec K_{m}$ differ at exactly $\left|I_{1}\right|$ vertices of $K_{n}$, indexed by $I_{1}$, the last contribution in the Kronecker product is an $n \times n$ matrix, whose entry $\left(x_{i}, x_{k}\right)$ must be equal to the distance $d(u, v)$. Then the claim follows from Proposition 3.1.

As in the case of the adjacency matrix $A d_{n} \prec A d_{m}$, the spectrum of the matrix $D$ can be computed by using a reduction argument. In fact, the matrix $D$ in (3.6) has the following block circulant structure

$$
D=\left(\begin{array}{ccccc}
D_{0} & D_{1} & D_{2} & \cdots & D_{m-1} \\
D_{m-1} & D_{0} & D_{1} & \ddots & \vdots \\
\vdots & D_{m-1} & \ddots & \ddots & \vdots \\
\vdots & & \ddots & \ddots & D_{1} \\
D_{1} & D_{2} & \cdots & D_{m-1} & D_{0}
\end{array}\right)
$$


with

$$
\begin{aligned}
D_{0} & =\sum_{\left(i_{2}, \ldots, i_{n}\right) \in\{0,1\}^{n}} A d_{m}^{i_{2}} \otimes \cdots \otimes A d_{m}^{i_{n}} \otimes A_{0, i_{2}, \ldots, i_{n}} \\
D_{i} & =\sum_{\left(i_{2}, \ldots, i_{n}\right) \in\{0,1\}^{n}} A d_{m}^{i_{2}} \otimes \cdots \otimes A d_{m}^{i_{n}} \otimes A_{1, i_{2}, \ldots, i_{n}} \quad \text { for each } i=1, \ldots, m-1 .
\end{aligned}
$$

Then the spectral analysis of block circulant matrices developed in [35] ensures that the spectrum of $D$ can be obtained by taking the union of the spectra of the following $m$ matrices of size $n m^{n-1}$ :

$$
\begin{aligned}
\widetilde{D}^{j_{1}}= & \sum_{h_{1}=0}^{m-1} \rho^{h_{1} j_{1}} D_{h_{1}} \\
= & \sum_{\left(i_{2}, \ldots, i_{n}\right) \in\{0,1\}^{n}} A d_{m}^{i_{2}} \otimes \cdots \otimes A d_{m}^{i_{n}} \otimes A_{0, i_{2}, \ldots, i_{n}}+ \\
& \quad+\sum_{h_{1}=1}^{m-1} \rho^{h_{1} j_{1}} \sum_{\left(i_{2}, \ldots, i_{n}\right) \in\{0,1\}^{n}} A d_{m}^{i_{2}} \otimes \cdots \otimes A d_{m}^{i_{n}} \otimes A_{1, i_{2}, \ldots, i_{n}} \\
= & \sum_{\left(i_{2}, \ldots, i_{n}\right) \in\{0,1\}^{n}} A d_{m}^{i_{2}} \otimes \cdots \otimes A d_{m}^{i_{n}} \otimes\left(A_{0, i_{2}, \ldots, i_{n}}+\sum_{h_{1}=1}^{m-1} \rho^{h_{1} j_{1}} A_{1, i_{2}, \ldots, i_{n}}\right),
\end{aligned}
$$

with $j_{1} \in\{0,1, \ldots, m-1\}$. Observe that each of these matrices is still a block circulant matrix, with blocks of size $n m^{n-2}$, given by

$$
\begin{aligned}
& D_{0}^{\prime}=\sum_{\left(i_{3}, \ldots, i_{n}\right) \in\{0,1\}^{n}} A d_{m}^{i_{3}} \otimes \cdots \otimes A d_{m}^{i_{n}} \otimes\left(A_{0,0, i_{3}, \ldots, i_{n}}+\sum_{h_{1}=1}^{m-1} \rho^{h_{1} j_{1}} A_{1,0, i_{3}, \ldots, i_{n}}\right), \\
& D_{i}^{\prime}=\sum_{\left(i_{3}, \ldots, i_{n}\right) \in\{0,1\}^{n}} A d_{m}^{i_{3}} \otimes \cdots \otimes A d_{m}^{i_{n}} \otimes\left(A_{0,1, i_{3}, \ldots, i_{n}}+\sum_{h_{1}=1}^{m-1} \rho^{h_{1} j_{1}} A_{1,1, i_{3}, \ldots, i_{n}}\right)
\end{aligned}
$$

for $i=1, \ldots, m-1$. Therefore, the same argument can be repeated, so that the spectrum of $D$ is obtained by taking the union of the spectra of the following $m^{2}$ matrices of size $n m^{n-2}$ :

$$
\begin{aligned}
\widetilde{D}^{j_{1}, j_{2}}= & \sum_{h_{2}=0}^{m-1} \rho^{h_{2} j_{2}} D_{h_{2}}^{\prime}= \\
=\sum_{\left(i_{3}, \ldots, i_{n}\right) \in\{0,1\}^{n}} A d_{m}^{i_{3}} \otimes \cdots \otimes A d_{m}^{i_{n}} \otimes & \left(A_{0,0, i_{3}, \ldots, i_{n}}+\sum_{h_{1}=1}^{m-1} \rho^{h_{1} j_{1}} A_{1,0, i_{3}, \ldots, i_{n}}\right)+ \\
& +\sum_{h_{2}=1}^{m-1} \rho^{h_{2} j_{2}} \sum_{\left(i_{3}, \ldots, i_{n}\right) \in\{0,1\}^{n}} A d_{m}^{i_{3}} \otimes \cdots \otimes d_{m}^{i_{n}} \otimes \\
& \otimes\left(A_{0,1, i_{3}, \ldots, i_{n}}+\sum_{h_{1}=1}^{m-1} \rho^{h_{1} j_{1}} A_{1,1, i_{3}, \ldots, i_{n}}\right)
\end{aligned}
$$


A. Donno: Spectrum, distance spectrum, and Wiener index of wreath products of ...

$$
\begin{aligned}
&=\sum_{\left(i_{3}, \ldots, i_{n}\right) \in\{0,1\}^{n}} A d_{m}^{i_{3}} \otimes \cdots \otimes A d_{m}^{i_{n}} \otimes\left(A_{0,0, i_{3}, \ldots, i_{n}}+\right. \\
&+\sum_{h_{1}=1}^{m-1} \rho^{h_{1} j_{1}} A_{1,0, i_{3}, \ldots, i_{n}}+\sum_{h_{2}=1}^{m-1} \rho^{h_{2} j_{2}} A_{0,1, i_{3}, \ldots, i_{n}}+ \\
&\left.+\sum_{h_{1}=1}^{m-1} \rho^{h_{1} j_{1}} \sum_{h_{2}=1}^{m-1} \rho^{h_{2} j_{2}} A_{1,1, i_{3}, \ldots, i_{n}}\right)
\end{aligned}
$$

with $\left(j_{1}, j_{2}\right) \in\{0, \ldots, m-1\}^{2}$. This reduction argument can be iterated further, until we get blocks of size $n$. Once again, notice that $\sum_{h=1}^{m-1} \rho^{h j_{s}}=\left\{\begin{array}{ll}-1 & \text { if } j_{s} \neq 0 \\ m-1 & \text { if } j_{s}=0 .\end{array}\right.$ We thus have proven the following theorem.

Theorem 3.11. The distance spectrum $\Sigma$ of the graph $K_{n} \prec K_{m}$ is obtained by taking the union of the spectra $\Sigma_{j_{1}, \ldots, j_{n}}$ of the $m^{n}$ matrices of size $n$ :

$$
\begin{aligned}
\widetilde{D}^{j_{1}, \ldots, j_{n}}= & \sum_{\left(i_{1}, \ldots, i_{n}\right) \in\{0,1\}^{n}} \prod_{s=1}^{n}\left(\sum_{h_{s}=1}^{m-1} \rho^{h_{s} j_{s}}\right)^{i_{s}} A_{i_{1}, \ldots, i_{n}}, \\
& \left(j_{1}, \ldots, j_{n}\right) \in\{0, \ldots, m-1\}^{n},
\end{aligned}
$$

where, if we put $I_{0}=\left\{i_{j}: i_{j}=0\right\}$ and $I_{1}=\left\{i_{j}: i_{j}=1\right\}$, we have:

$$
\begin{aligned}
A_{0, \ldots, 0} & =A d_{n} \\
A_{i_{1}, \ldots, i_{n}}\left(x_{i}, x_{k}\right) & =\left\{\begin{array}{ll}
1 & \text { if } i, k \in I_{1} \\
3 & \text { if } i, k \in I_{0} \\
2 & \text { if } i \in I_{1}, k \in I_{0} ; \text { or } i \in I_{0}, k \in I_{1}
\end{array} \quad \text { for }\left|I_{1}\right|=1 ;\right.
\end{aligned}
$$

and

$$
\begin{array}{r}
A_{i_{1}, \ldots, i_{n}}\left(x_{i}, x_{k}\right)= \begin{cases}2\left|I_{1}\right|+1 & \text { if } i, k \in I_{0} \\
2\left|I_{1}\right| & \text { if } i \in I_{0}, k \in I_{1} ; i \in I_{1}, k \in I_{0} \\
2\left|I_{1}\right|-1+\delta_{i k} & \text { if } i, k \in I_{1}\end{cases} \\
\text { for } 2 \leq\left|I_{1}\right| \leq n, \text { with } \delta_{i k}= \begin{cases}1 & \text { if } i=k \\
0 & \text { if } i \neq k .\end{cases}
\end{array}
$$

Example 3.12. Let us consider the explicit example $K_{3} \succ K_{3}$. The distance matrix of this graph is

$$
\begin{array}{r}
D=I_{3} \otimes I_{3} \otimes I_{3} \otimes A_{000}+I_{3} \otimes I_{3} \otimes A d_{3} \otimes A_{001}+I_{3} \otimes A d_{3} \otimes I_{3} \otimes A_{010}+ \\
+I_{3} \otimes A d_{3} \otimes A d_{3} \otimes A_{011}+A d_{3} \otimes I_{3} \otimes I_{3} \otimes A_{100}+A d_{3} \otimes I_{3} \otimes A d_{3} \otimes A_{101}+ \\
+A d_{3} \otimes A d_{3} \otimes I_{3} \otimes A_{110}+A d_{3} \otimes A d_{3} \otimes A d_{3} \otimes A_{111},
\end{array}
$$

with

$$
A_{000}=\left(\begin{array}{ccc}
0 & 1 & 1 \\
1 & 0 & 1 \\
1 & 1 & 0
\end{array}\right) \quad A_{001}=\left(\begin{array}{ccc}
3 & 3 & 2 \\
3 & 3 & 2 \\
2 & 2 & 1
\end{array}\right)
$$




$$
\begin{aligned}
A_{010}=\left(\begin{array}{lll}
3 & 2 & 3 \\
2 & 1 & 2 \\
3 & 2 & 3
\end{array}\right) & A_{011}=\left(\begin{array}{lll}
5 & 4 & 4 \\
4 & 4 & 3 \\
4 & 3 & 4
\end{array}\right) \\
A_{100}=\left(\begin{array}{lll}
1 & 2 & 2 \\
2 & 3 & 3 \\
2 & 3 & 3
\end{array}\right) & A_{101}=\left(\begin{array}{lll}
4 & 4 & 3 \\
4 & 5 & 4 \\
3 & 4 & 4
\end{array}\right) \\
A_{110}=\left(\begin{array}{lll}
4 & 3 & 4 \\
3 & 4 & 4 \\
4 & 4 & 5
\end{array}\right) & A_{111}=\left(\begin{array}{lll}
6 & 5 & 5 \\
5 & 6 & 5 \\
5 & 5 & 6
\end{array}\right) .
\end{aligned}
$$

The spectrum of $D$ consists of the following eigenvalues:

$$
312 ; 15^{2} ; 0^{48} ;(-3)^{18} ;(-24 \pm 3 \sqrt{43})^{6} .
$$

We have, for instance:

$$
\begin{array}{r}
\widetilde{D}^{0,2,0}=A_{000}+2 A_{001}-A_{010}-2 A_{011}+2 A_{100}+4 A_{101}-2 A_{110}-4 A_{111}= \\
=\left(\begin{array}{ccc}
-21 & -9 & -18 \\
-9 & -9 & -9 \\
-18 & -9 & -21
\end{array}\right)
\end{array}
$$

whose eigenvalues are -3 and $-24 \pm 3 \sqrt{43}$.

Next, we pass to the computation of the Wiener index $W\left(K_{n} \imath K_{m}\right)$ of the graph $K_{n}$ ح $K_{m}$. It follows from the definition of the Wiener index that $W\left(K_{n} \imath K_{m}\right)$ is given by the sum of all the entries of $D$, divided by 2 , due to the fact that each contribution $d(u, v)$ appears twice, as the matrix $D$ is symmetric. Keeping in mind the block structure of the distance matrix $D$ described in (3.7) and the fact that each block $D_{i}$, for $i=0, \ldots, m-1$, appearing in (3.7) can be recursively regarded as a block circulant matrix, until one gets elementary blocks of size $n$ represented by matrices of type $A_{i_{1}, \ldots, i_{n}}$, we obtain the following result.

Theorem 3.13. The Wiener index of the graph $K_{n} \prec K_{m}$ is

$$
W\left(K_{n} \curlywedge K_{m}\right)=\frac{n m^{n}}{2}\left(2 m^{n} n^{2}-n m^{n}-2 n^{2} m^{n-1}+m^{n}+2 n m^{n-1}-m^{n-1}-m\right) .
$$

Proof. First of all, for every $n$-tuple $\left(i_{1}, \ldots, i_{n}\right) \in\{0,1\}^{n}$, put:

$$
d_{i_{1}, \ldots, i_{n}}=\sum_{x_{i}, x_{j} \in V_{n}} A_{i_{1}, \ldots, i_{n}}\left(x_{i}, x_{j}\right) .
$$

Now observe that, by definition of the matrices $A_{i_{1}, \ldots, i_{n}}$, the sum $d_{i_{1}, \ldots, i_{n}}$ only depends on the cardinality of the sets $I_{0}=\left\{i_{j}: i_{j}=0\right\}$ and $I_{1}=\left\{i_{j}: i_{j}=1\right\}$, while it is independent from the particular position of the indices. Therefore, for every $k=0,1, \ldots, n$, it makes sense to define:

$$
d_{k}=\sum_{x_{i}, x_{j} \in V_{n}} A_{\underbrace{1, \ldots, 1}_{k \text { times }}}^{1, \ldots, \ldots, 0}(\underbrace{0, \ldots}_{n-k \text { times }}, x_{j}) .
$$

Moreover, by performing a direct computation which uses the explicit description of the matrices $A_{i_{1}, \ldots, i_{n}}$ given in Theorem 3.11, we are able to determine: 
(a) $d_{0}=n(n-1)$;

(b) $d_{1}=1+4(n-1)+3(n-1)^{2}$;

(c) $d_{k}=(2 k+1)(n-k)^{2}+4 k^{2}(n-k)+2 k^{2}+k(k-1)(2 k-1)$ for $2 \leq k \leq n$.

Now we have to establish the number of contributions of type $d_{k}$ to $W\left(K_{n} \imath K_{m}\right)$, for every $k$. First of all, a factor equal to $\left(\begin{array}{l}n \\ k\end{array}\right)$ appears, taking into account all the possible choices of $k$ indices equal to 1 . Moreover, a second factor given by $m^{n}(m-1)^{k}$ appears, since a fixed $n$-tuple $\left(\bar{i}_{1}, \ldots, \bar{i}_{n}\right)$ containing $k$ indices equal to 1 (see Equation (3.6)) produces $m^{n}(m-1)^{k}$ blocks of size $n$, within the matrix $D$, which are equal to $A_{\bar{i}_{1}, \ldots, \bar{i}_{n}}$, due to the fact that, when we change the color of a lamp, we have $m-1$ possibilities for the choice of the new color. This implies that

$$
W\left(K_{n} \curlywedge K_{m}\right)=\frac{m^{n}}{2} \sum_{k=0}^{n}\left(\begin{array}{l}
n \\
k
\end{array}\right)(m-1)^{k} d_{k} .
$$

By explicitly computing the sum in (3.8), we get the claim.

Example 3.14. Consider the case of $K_{3}$ 乙 $K_{3}$. Theorem 3.13 gives $W\left(K_{3} \prec K_{3}\right)=12636$, with $d_{0}=6 ; d_{1}=21 ; d_{2}=35 ; d_{3}=48$.

\section{References}

[1] N. Alon, A. Lubotzky and A. Wigderson, Semi-direct product in groups and zig-zag product in graphs: connections and applications, in: $42^{\text {nd }}$ IEEE Symposium on Foundations of Computer Science (Las Vegas, Nevada, 2001), IEEE Computer Soc., Los Alamitos, California, pp. 630 637, 2001, doi:10.1109/SFCS.2001.959939.

[2] R. A. Bailey, C. E. Praeger, C. A. Rowley and T. P. Speed, Generalized wreath products of permutation groups, Proc. London Math. Soc. 47 (1983), 69-82, doi:10.1112/plms/s3-47.1.69.

[3] L. Bartholdi and W. Woess, Spectral computations on lamplighter groups and Diestel-Leader graphs, J. Fourier Anal. Appl. 11 (2005), 175-202, doi:10.1007/s00041-005-3079-0.

[4] A. E. Brouwer, A. M. Cohen and A. Neumaier, Distance-Regular Graphs, volume 18 of Ergebnisse der Mathematik und ihrer Grenzgebiete, Springer-Verlag, Berlin, 1989, doi: 10.1007/978-3-642-74341-2.

[5] A. E. Brouwer and W. H. Haemers, Spectra of Graphs, Universitext, Springer-Verlag, New York, 2012, doi:10.1007/978-1-4614-1939-6.

[6] D. D'Angeli and A. Donno, Isomorphism classification of infinite Sierpiński carpet graphs, AIP Conf. Proc. 1648 (2015), 570002, doi:10.1063/1.4912788, proceedings of the International Conference on Numerical Analysis and Applied Mathematics 2014.

[7] D. D'Angeli and A. Donno, Metric compactification of infinite Sierpiński carpet graphs, Discrete Math. 339 (2016), 2693-2705, doi:10.1016/j.disc.2016.04.023.

[8] D. D'Angeli and A. Donno, Wreath product of matrices, Linear Algebra Appl. 513 (2017), 276-303, doi:10.1016/j.laa.2016.10.023.

[9] D. D'Angeli, A. Donno and A. Monti, Computing the Wiener index in Sierpiński carpet graphs, AIP Conf. Proc. 1738 (2016), 270008, doi:10.1063/1.4952047, proceedings of the International Conference on Numerical Analysis and Applied Mathematics 2015.

[10] D. D'Angeli, A. Donno and E. Sava-Huss, Connectedness and isomorphism properties of the zig-zag product of graphs, J. Graph Theory 83 (2016), 120-151, doi:10.1002/jgt.21917. 
[11] P. J. Davis, Circulant Matrices, Pure and Applied Mathematics, John Wiley \& Sons, New York, Chichester, Brisbane, Toronto, 1979.

[12] A. A. Dobrynin, R. Entringer and I. Gutman, Wiener index of trees: theory and applications, Acta Appl. Math. 66 (2001), 211-249, doi:10.1023/a:1010767517079.

[13] A. A. Dobrynin, I. Gutman, S. Klavžar and P. Žigert, Wiener index of hexagonal systems, Acta Appl. Math. 72 (2002), 247-294, doi:10.1023/a:1016290123303.

[14] A. Donno, Replacement and zig-zag products, Cayley graphs and Lamplighter random walk, Int. J. Group Theory 2 (2013), 11-35, http://ijgt.ui.ac.ir/article_1932. html.

[15] A. Donno, Generalized wreath products of graphs and groups, Graphs Combin. 31 (2015), 915-926, doi:10.1007/s00373-014-1414-4.

[16] A. Donno and D. Iacono, Distances and isomorphisms in 4-regular circulant graphs, AIP Conf. Proc. 1738 (2016), 270002, doi:10.1063/1.4952041, proceedings of the International Conference on Numerical Analysis and Applied Mathematics 2015.

[17] M. Eliasi, G. Raeisi and B. Taeri, Wiener index of some graph operations, Discrete Appl. Math. 160 (2012), 1333-1344, doi:10.1016/j.dam.2012.01.014.

[18] M. Eliasi and B. Taeri, Four new sums of graphs and their Wiener indices, Discrete Appl. Math. 157 (2009), 794-803, doi:10.1016/j.dam.2008.07.001.

[19] A. Erschler, Generalized wreath products, Int. Math. Res. Notices 2006 (2006), 57835, doi: 10.1155/imrn/2006/57835.

[20] Ł. Grabowski, On Turing dynamical systems and the Atiyah problem, Invent. Math. 198 (2014), 27-69, doi:10.1007/s00222-013-0497-5.

[21] R. I. Grigorchuk and A. Żuk, The lamplighter group as a group generated by a 2-state automaton, and its spectrum, Geom. Dedicata 87 (2001), 209-244, doi:10.1023/a:1012061801279.

[22] R. Hammack, W. Imrich and S. Klavžar, Handbook of Product Graphs, Discrete Mathematics and its Applications, CRC Press, Boca Raton, Florida, 2nd edition, 2011, doi:10.1201/b10959.

[23] W. Imrich and H. Izbicki, Associative products of graphs, Monatsh. Math. 80 (1975), 277-281, doi:10.1007/BF01472575.

[24] G. Indulal, Distance spectrum of graph compositions, Ars Math. Contemp. 2 (2009), 93-100, http://amc-journal.eu/index.php/amc/article/view/103.

[25] G. Indulal and I. Gutman, On the distance spectra of some graphs, Math. Commun. 13 (2008), 123-131, http://hrcak.srce.hr/23569.

[26] F. Lehner, On the eigenspaces of lamplighter random walks and percolation clusters on graphs, Proc. Amer. Math. Soc. 137 (2009), 2631-2637, doi:10.1090/S0002-9939-09-09869-4.

[27] F. Lehner, M. Neuhauser and W. Woess, On the spectrum of lamplighter groups and percolation clusters, Math. Ann. 342 (2008), 69-89, doi:10.1007/s00208-008-0222-7.

[28] J. D. P. Meldrum, Wreath Products of Groups and Semigroups, volume 74 of Pitman Monographs and Surveys in Pure and Applied Mathematics, Longman, Harlow, 1995.

[29] O. Reingold, S. Vadhan and A. Wigderson, Entropy waves, the zig-zag graph product, and new constant-degree expanders, Ann. of Math. 155 (2002), 157-187, doi:10.2307/3062153.

[30] G. Sabidussi, The composition of graphs, Duke Math. J 26 (1959), 693-696, doi:10.1215/ S0012-7094-59-02667-5.

[31] G. Sabidussi, Graph multiplication, Math. Z. 72 (1959), 446-457, doi:10.1007/bf01162967.

[32] F. Scarabotti and F. Tolli, Harmonic analysis of finite lamplighter random walks, J. Dyn. Control Syst. 14 (2008), 251-282, doi:10.1007/s10883-008-9038-8. 
[33] F. Scarabotti and F. Tolli, Harmonic analysis on a finite homogeneous space, Proc. London Math. Soc. 100 (2010), 348-376, doi:10.1112/plms/pdp027.

[34] D. Stevanović and G. Indulal, The distance spectrum and energy of the compositions of regular graphs, Appl. Math. Lett. 22 (2009), 1136-1140, doi:10.1016/j.aml.2008.11.007.

[35] G. J. Tee, Eigenvectors of block circulant and alternating circulant matrices, New Zealand J. Math. 36 (2007), 195-211, http://nzjm.math.auckland.ac.nz/index. php/Eigenvectors_of_Block_Circulant_and_Alternating_Circulant_ Matrices.

[36] H. Wiener, Structural determination of paraffin boiling points, J. Am. Chem. Soc. 69 (1947), 17-20, doi:10.1021/ja01193a005.

[37] W. Woess, A note on the norms of transition operators on lamplighter graphs and groups, Internat. J. Algebra Comput. 15 (2005), 1261-1272, doi:10.1142/S0218196705002591. 